\title{
The ion beam sputtering facility at KURRI: coatings for advanced neutron optical devices
}

\author{
Masahiro Hino ${ }^{\text {a }}$, Tatsuro Oda ${ }^{\mathrm{b}}$, Masaaki Kitaguchi ${ }^{\mathrm{c}}$, Norifumi L. Yamada ${ }^{\mathrm{d}}$, \\ Seiji Tasaki ${ }^{\mathrm{b}}$ and Yuji Kawabata ${ }^{\mathrm{a}}$
}

\author{
${ }^{a}$ Research Reactor Institute, Kyoto university, Kumatori,Osaka, 590-0494, Japan, \\ ${ }^{\mathrm{b}}$ Department of Nuclear Engineering, Kyoto University, Kyoto, 615-8540, Japan \\ ${ }^{\mathrm{c}}$ Center for Experimental Studies, KMI, Nagoya University, Nagoya 464-8602, Japan, \\ ${ }^{\mathrm{d}}$ Neutron Science Laboratory, High Energy Accelerator Research Organization, 203-1 Shirakata, Tokai, \\ Ibaraki, 319-1106, Japan
}

\begin{abstract}
We describe a film coating facility for the development of multilayer mirrors for use in neutron optical devices that handle slow neutron beams. Recently, we succeeded in fabricating a large neutron supermirror with high reflectivity using an ion beam sputtering system (KUR-IBS), as well as all neutron supermirrors in two neutron guide tubes at BL06 at J-PARC/MLF. We also realized a large flexible self-standing $m=5 \mathrm{NiC} / \mathrm{Ti}$ supermirror and very small $d$-spacing $(d=1.65 \mathrm{~nm})$ multilayer sheets. In this paper, we present an overview of the performance and utility of non-magnetic neutron multilayer mirrors fabricated with the KUR-IBS
\end{abstract}

\section{Introduction}

2 The slow neutron beam is a very powerful probe for material science, life science, and industrial applications. The 3 demand for neutron beams in various research fields continues to increase, and large-scale neutron beam facilities 4 based on proton accelerators, such as the J-PARC in Japan and SNS in USA, have been constructed; meanwhile, 5 Europe and China have launched the ESS and CSNS, respectively. Small-scale neutron source development is also active [1]. Even at the large-scale facilities, the intensity and brilliance of the neutron source is still quite weak in comparison to X-rays from synchrotron radiation. As a result, high-performance neutron optical devices are very 8 important, and the multilayer mirror is one of the most important devices for neutron beam handling. The multilayer 9 mirror consists of alternating layers of two materials with different potential energies for the neutron. It has artificial 10 lattice spacing ( $d$-spacing) that produces one-dimensional optical potential for a neutron beam. Development of 11 multilayer mirrors is still being widely conducted for application in bending, polarizing, shaping and focusing neutron 12 beams. A supermirror is a stack of multilayer mirrors with gradually increasing $d$-spacing values. The supermirror is 13 widely used as key device in the neutron guide tube [2]. To the best of our knowledge, the world's first full-scale 14 supermirror neutron guide tube was constructed in 1985 at the Kyoto University Research Reactor Institute (KURRI), 15 [3].

\footnotetext{
* Corresponding author. Tel.: +81 724512450

E-mail address: hino@rri.kyoto-U.ac.jp (M. Hino)
} 
17 There are two kinds of PVD coating systems for fabrication of neutron optical devices at the KURRI. One is based on a 18 vacuum deposition technique [4], and the other is ion beam sputtering (IBS) [5]. A multilayer with small $d$-spacing and 19 supermirror with large- $m$ are desirable for enlarging utilization efficiency in neutron scattering experiments. Here, $m$ is 20 the maximum critical angle of the mirror in units of critical angle of natural nickel. The required number of layers is at 21 least proportional to the fourth power of $m$, according to Porod's law. The IBS technique enables us to fabricate smooth layer structures with sharp edges. In addition, the adhesion force to the substrate with IBS deposition is much larger than with vacuum deposition. In fact, the neutron optics group the Japan Atomic Energy Agency (JAEA) installed a large-scale IBS system for neutron spectrometers at J-PARC/MLF [6-8], and succeeded in fabricating a large-scale neutron supermirror with high reflectivity and low diffuse scattering even on a plano-elliptical glass substrate [9-10]. The effective substrate area at the JAEA-IBS system is $500 \mathrm{~mm}$ in diameter. It is possible to fabricate a lot of supermirrors in a neutron guide tube and focusing mirror. On the other hand, the maximum substrate area at the KUR-IBS was limited to $200 \mathrm{~mm}$ in diameter, and the effective substrate area was smaller than the designed value due to the small ion gun size.

In general, DC magnetron sputter is popular for the production of neutron multilayer mirrors, since it is possible to produce large sizes at reasonable cost with high throughput deposition [11-17]. The smaller size was particularly limited in actual application. Recently, we succeeded in enlarging the effective deposition size without significantly modifying the KUR-IBS. The throughput deposition rate was almost the same as that in our previous work [5, 18], and production costs were also reasonable. In addition, we recently successfully fabricated all neutron supermirrors in two neutron guide tubes using the KUR-IBS, the total length of which was $29 \mathrm{~m}$ at BL06 at J-PARC/MLF [19]. In this study, we present an overview of the performance and utility of the non-magnetic neutron multilayer mirrors fabricated by the KUR-IBS.

\section{Multilayer fabrication system and the performance of small-size multilayers}

Figure 1 shows the schematic structure of the KUR-IBS coating system, in which the outlet of the ion gun is $12 \mathrm{~cm}$ in diameter. The system is a special modified version of the Veeco IBD-350. The ion gun is generally used to generate $\mathrm{Ar}^{+}$ions. The accelerator voltage and current of $\mathrm{Ar}^{+}$ions can be controlled with the resolution of $1 \mathrm{~V}$ and $1 \mathrm{~mA}$, and typical values were from 120 to $240 \mathrm{~mA}$, respectively. The deposition rate is proportional to the current, and it is necessary to use high current for high throughput. However, a typical current was used with 120 or $150 \mathrm{~mA}$, since the smoothness of the layer and service life of the ion gun are better with low-current operation. There are two kinds of vacuum pumps, the turbo-molecular pump and cryo-pump shown in Fig. 1. The outer size of the process chamber was $1.2 \mathrm{~m}$ wide $\times 0.9 \mathrm{~m}$ long $\times 1.1 \mathrm{~m}$ high. That doesn't include the ion gun system, vacuum pumps, stand, lord lock chamber, etc.; the process chamber itself was thus not huge. It could reach up to $5 \times 10^{-6} \mathrm{~Pa}$ vacuum degrees. In general use, the base pressure before deposition was $5 \times 10^{-5} \mathrm{~Pa}$, and that during deposition was $1.3 \times 10^{-2} \mathrm{~Pa}$. It took half a day to vacuum up to base pressure from atmospheric pressure. The deposition rate and quality of the layer also depended on 
the tilt angle of the substrate holder, $\phi$. In the case of a small multilayer, it was possible to deposit multilayers on five consecutive wafers without breaking the vacuum of chamber using lord-lock system. The substrate was placed at the rotation center of the substrate holder with a tilt angle $\phi$ of -65 degrees. It was possible for the substrate holder to move with the tilt angle $\phi$ from 90 to -80 degrees, and to rotate up to 10 turns a min. Here, the tilt angle $\phi=0$ corresponds to the vertical direction, and the isolation valve between the process chamber and lord lock chamber could open at an angle of 90 degrees. The angles of targets were fixed. It was possible to use 6 targets, and the switching time was less than $20 \mathrm{~s}$. The beam spot size at the target could be controlled. The typical deposition rate of $\mathrm{NiC}$ and $\mathrm{Ti}$ targets were 0.03 and $0.035 \mathrm{~nm} / \mathrm{s}$, and the each layer thickness was controlled by deposition time with constant accelerator voltage and current. The minimum time resolution was 1 millisecond using an electric shutter.

Figure 2 shows reflectivity of a small $d$-spacing NiC/Ti multilayer mirror deposited on the same silicon wafer, and the cross-sectional TEM image taken by Dr. Thomas Krist's group in HZB. In the TEM image, the black and white lines indicate NiC and Ti layers, respectively. The reflectivity was measured with a MINE2 reflectometer at JRR-3 at JAEA. The incident neutron wavelength was $0.88 \mathrm{~nm}$, the resolution was $2.7 \%$ in FWHM, and the divergent angle was smaller than 1 milliradian. The designed $d$-spacing and thickness distribution were $2.9 \mathrm{~nm}$ and $5 \%$, respectively. The $d$-spacing of $2.9 \mathrm{~nm}$ corresponds to the critical angle of an $m=10$ supermirror, and the distribution was determined in order to reflect all cold neutron beams at the MINE2. The layer thickness was gradually changed by controlling the deposition time, and the measured reflectivity was well reproduced by the theoretical line with the average $d$-spacing of $2.9 \mathrm{~nm}$. The theoretical line was calculated by a one-dimensional Schrödinger equation, including the effect of a surface roughness of Debye-Waller factor of $0.55 \mathrm{~nm}$. Here the nuclear potential values of $\mathrm{NiC}$ and Ti layers were ideal: 243 and $-51 \mathrm{neV}$, respectively.

In order to fabricate a large- $m$ supermirror and wide-band small $d$-spacing multilayer with high reflectivity, even with a theoretically smooth surface and ideal layer structure, the required number of layers increases to the fourth power of $m$. The surface and interface roughness of the multilayer increases with total thickness, and we tested the performance of a stacking multilayer technique. It enabled us to realize very small $d$-spacing and wide-band monochromator, as shown in Fig. 3(a). The $d$-spacing at maximum intensity was $2.89 \mathrm{~nm}$, and the reflectivity was higher than 0.4 and was actually used as a key device of a Doppler shifter for the production of ultra-cold neutrons [20]. The total number of layers for the monochromator was 85060. The reflectivity was measured with the MINE2 reflectometer at JRR-3 at JAEA. The monochromator consisted of four mirrors. Each was a thin silicon substrate, 0.3-mm thick, coated on both surfaces. In other words, each mirror consisted of two multilayers and the thin silicon substrate. For the mirrors, the first and second multilayers were deposited on the incident and backside of the silicon wafer to reflect the neutron beam. The numbers of layers on the first and second multilayers were 10336 and 10929, respectively. Figure 3(b) shows the reflectivities of the first and second mirrors, as well as that of a two-mirror stack. The figure clearly shows that both the reflectivity and band-width were increased by stacking the different $d$-spacing multilayers. 
83 Figure 4 shows measured reflectivity by an $m=7 \mathrm{NiC} / \mathrm{Ti}$ supermirror, which consisted of two stacked mirrors. The layer

84 sequence was designed using an algorithm based on Pleshanov [21]. Each mirror also consisted of two multilayers. The 85 first multilayer was an $m=5 \mathrm{NiC} / \mathrm{Ti}$ supermirror deposited on a thin silicon wafer $0.3-\mathrm{mm}$ thick. The number of layers 86 was 4766 . The layer thickness of the first multilayer ( $m=5$ supermirror) was gradually increased from the silicon 87 surface. The second multilayer was a wide-band multilayer deposited on the backside of the thin silicon wafer. The 88 design $d$-spacing was from 5.82 to $4.76 \mathrm{~nm}$, and the total number of layers was 7131 . The layer thickness was gradually 89 decreased from the silicon surface. The reflectivity of the first mirror featured a big valley, around $m=5.5$, and 90 reflectivity loss was significantly improved by stacking the two mirrors. These results indicate that the stacking 91 technique is useful for a small number of special uses. It is not easy to achieve production of large amounts for using in 92 a neutron guide tube. It is more effective for use with very small $d$-spacing mirrors with wide bands; the influence of 93 the substrate is not negligible with an increase in the number of stacking mirrors and self-standing multilayers like 94 crystal is one of the ultimate goals.

\section{3. Production of large multilayer neutron mirrors}

96 In section 2, we showed the high performance and utility of non-magnetic neutron multilayer mirrors fabricated with 97 the KUR-IBS. However, the maximum wafer size and thickness were limited to 200 and $3 \mathrm{~mm}$, respectively. The small 98 size problem was really limiting the application. Size was limited by the load lock system and ion gun size. 99 Geometrically, the process vacuum chamber could accept insertion of discs up to $500 \mathrm{~mm}$ in diameter. We decided to 100 stop using the lord lock system and developed a new substrate holding system. The outlet of the ion gun size was about $10112 \mathrm{~cm}$ in diameter, and the thickness of the uniform area was almost the same as the outlet size. Figure 5(a) and 5(b) 102 are schematic illustrations of the thickness dependence of the tilt angles $\phi=-65$ and $\phi=-17$ degrees, respectively. As 103 shown in Fig. 5(a), it was not necessary to rotate the substrate holder for small samples, while 5(b) shows that the 104 coating for a large sample was impossible without this rotation. The area of uniform thickness with $\phi=-17$ was smaller 105 than that with $\phi=-65$ degrees; however, the thickness dependence is similar to a triangle, as shown in Fig. 5(c).

106 In the cross-sectional geometrical view, it is possible to fabricate large multilayer mirrors by rotating the substrate 107 holder. When we introduced a collimator between the target and the substrate holder, we could get a larger uniform 108 layer; however, the throughput was dramatically reduced. We aimed to demonstrate that actual production of large-size 109 neutron mirrors is feasible even with the KUR-IBS, in which ion gun is not so large. In fact, large-size neutron 110 supermirrors were fabricated without any collimator by adjusting the deposition parameters. The layer thickness was 111 controlled by deposition time, and the current with constant voltage; the minimum time and current resolution were 10 $112 \mathrm{~s}$ and $1 \mathrm{~mA}$, respectively. The time resolution was determined by the period of rotation of the substrate holder to obtain 113 uniform thickness in each layer. The new substrate holder moved with the tilt angle $\phi=-17$ deg, and rotated 6 turns a $114 \mathrm{~min}$. The typical deposition rates of the $\mathrm{NiC}$ and $\mathrm{Ti}$ targets were 0.02 and $0.03 \mathrm{~nm} / \mathrm{s}$, and the accelerator voltage and 115 current of the ion gun were $800 \mathrm{~V}$ and $150 \mathrm{mV}$, respectively. It took $1,2,3$, and 5 days for fabrication of $m=2,2.5,3$, 116 and $4 \mathrm{NiC} / \mathrm{Ti}$ supermirrors, respectively, at one batch including vacuum pumping. Figure 6 shows reflectivities of the 
$m=4 \mathrm{NiC}$ supermirror at a batch. As shown in the image insert in Fig. 6, four $m=4 \mathrm{NiC} / \mathrm{Ti}$ supermirrors were deposited

118 on smooth glass $300 \mathrm{~mm}$ long x $70 \mathrm{~mm}$ wide. The reflectivities were measured with a SOFIA reflectometer at 119 J-PARC/MLF [22, 23]. The reflectivities at all points were high, although the critical angle of total reflection differed 120 little by position. Thus, we enlarged the effective deposition area to more than five times the previous condition and 121 successfully fabricated all neutron supermirrors in two neutron guide tubes of BL06 at J-PARC. These reflectivities 122 were measured and their performance was high [19].

\section{4. Large flexible self-standing multilayer sheet}

124 In the previous section, we clearly demonstrated that a large-size multilayer mirror can be fabricated even with a small 125 ion gun. This technique enables us to fabricate supermirrors on curved surfaces for ellipsoidal focusing mirrors. 126 Recently, forming and polishing techniques for curved substrates have been dramatically improved [25, 26]; however, 127 it is still very difficult to develop a large- $m$ and small $d$-spacing multilayer mirror because it takes a long time to 128 fabricate and the coatings can easily peel off from the substrate due to the increase of internal stress of the multilayer. 129 The stress increases with total thickness, and sometimes destroys the surface of the glass substrate. From the point of 130 view of simple material mechanics, the stiffness of the multilayer is proportional to the third power of the total 131 thickness. This implies that a large- $m$ supermirror and small $d$-spacing multilayer mirror are feasible owing to the thick 132 total thickness. In fact, the total thickness of an $m=5 \mathrm{NiC} / \mathrm{Ti}$ supermirror is larger than $10 \mu \mathrm{m}$, and it is easy handling 133 the shape compared to a small- $m$ supermirror. As shown in Fig. 7(a) and (b), large-size self-standing $m=5 \mathrm{NiC} / \mathrm{Ti}$ 134 supermirror sheets were peel off from the glass substrate without the breaking by controlling the adhesion force 135 between the glass surface and thickest $\mathrm{NiC}$ layer of supermirror. The concept of peeling technique is similar to 136 production of replica mirrors [27, 28]. The mirrors are copied from plane or cylindrical glass surfaces in the following 137 way: First, Ni monolayer or Ni/Ti multilayer is deposited on the glass substrates. Second, nickel or copper electroplated 138 on these films as a supporting foil in which thickness is thicker than $0.15 \mathrm{~mm}$. This composite metal sheet can 139 subsequently be removed from the glass substrate. On the other hand, the self-standing sheet has no supporting foil and 140 consists of neutron multilayer mirror itself. As shown in Fig.7 (c), neutron flux can be increased by concentrically 141 nesting mirrors with the same focal length and curvature. It is one of ultimate goals for development of self-standing 142 large- $m$ supermirror sheet. Here winding direction of the sheet is very important in order to approximate the required 143 shape. The incident neutrons were reflected by the inner side of the winding sheet and the inner and outer surface 144 should be the thickest NiC layer and thinnest $d$-spacing, respectively. In Fig.7 (b), the winding direction was controlled 145 in the right direction for the use of the nesting mirrors. We cut $5 \mathrm{~cm}$ squares from the outer circumference of the sheet 146 and affixed them on the glass plate with thin double-sided tape, $0.01 \mathrm{~mm}$ thick. As shown in Fig. 8, the reflectivity even 147 at the outer circumference was nearly the same as that of an ordinary flat supermirror on the glass substrate. It is 148 possible to put the layer on the inner surface of an ellipsoidal shape; however, it is still very difficult to control the waviness, and it is necessary to improve the forming fixation for the use of axisymmetric focusing devices.

There are some applications for VCN experiments without the improvement, since the required form accuracy is not so severe. In particular, this is a very promising device for the blade mirror of a Doppler shifter [20]. The incident angle to 
152 the mirror is almost 90 degrees, and the waviness of the mirror is negligible. A smaller $d$-spacing wide-band mirror is 153 required in the use of an intense neutron wavelength. The incident neutron velocity corresponds to the rotating speed of 154 the mirror, and the weight of the mirror should be light. Fig. 9 shows the reflectivity of a self-standing very small $155 d$-spacing multilayer in which the layers numbered 10000. A sharp Bragg peak can clearly be observed at $\mathrm{Q}_{z}=3.8 \mathrm{~nm}^{-1}$, 156 although the reflectivity $\left(1.5 \times 10^{-3}\right)$ was low. The low reflectivity is estimated with interfacial roughness of $0.7 \mathrm{~nm}$.

157 The sputtering condition for large size was not optimized for the thinnest d-spacing. In the part cut off from the outer 158 circumference of the sheet, the effective deposition rate is high. Ni crystal was easy to grow and NiC layer was not 159 smooth layer. In fact, we observed relatively strong diffuse scattering. The surface roughness of the glass substrate was 160 not confirmed for the thinnest d-spacing. We are improving the reflectivity for practical use by optimizing sputtering 161 and substrate conditions for the thinnest d-spacing.

\section{Conclusion}

164 We described the ion beam sputtering system at Kyoto University Reactor (KUR-IBS) and the performance of 165 fabricated non-magnetic multilayer mirrors. In the case of initial maximum design of a deposition area smaller than 200 $166 \mathrm{~mm}$ in diameter, the $m=7 \mathrm{NiC} / \mathrm{Ti}$ supermirror and wide-band small $d$-spacing $(d=2.9 \mathrm{~nm})$ multilayer with high 167 reflectivity were realized by a stacking technique.

168 The effective deposition area was enlarged to $460 \mathrm{~mm}$ in diameter and high-performance large-size neutron multilayer 169 mirrors were fabricated, even though the size of the KUR-IBS ion gun was much smaller than the deposition area. 170 Large flexible self-standing sheets of $m=5 \mathrm{NiC} / \mathrm{Ti}$ supermirror and very small $d$-spacing $(d=1.65 \mathrm{~nm})$ multilayer were 171 also realized. Although the application as a neutron-focusing device is still limited due to the waviness of the sheets, 172 the fabrication technique has been almost established and the throughput is high. The KUR-IBS is already more than 10 173 years old; however, it is still very useful and continues to be improved for the development of advanced neutron 174 multilayer mirrors. 


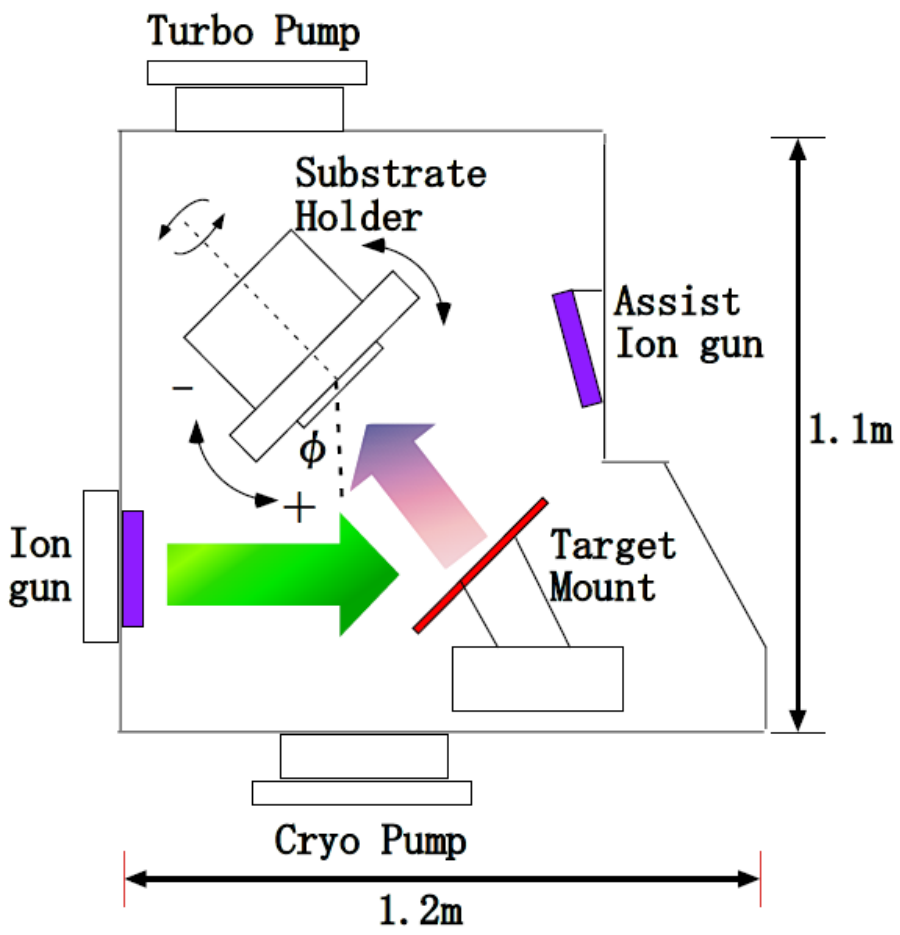

178 Fig. 1 Schematic view of ion beam sputtering system at KURRI.
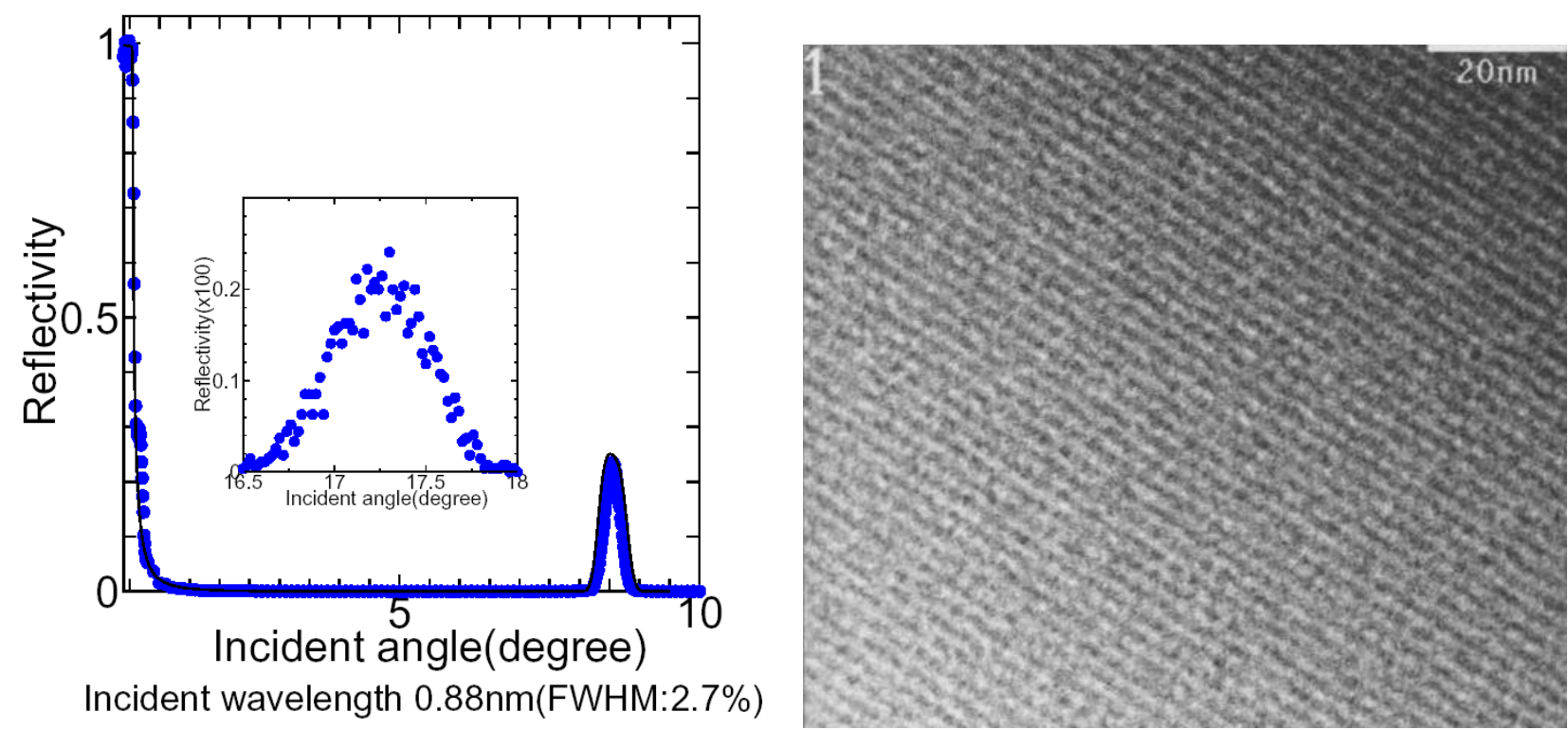

181 Fig. 2 Reflectivity of $d=2.89 \mathrm{~nm}$ NiC/Ti multilayer mirror with 10000 layers; the cross-sectional TEM image. The 182 inserted graph shows reflectivity of the second order. The incident neutron wavelength was $0.88 \mathrm{~nm}$. 
185

186 187

188
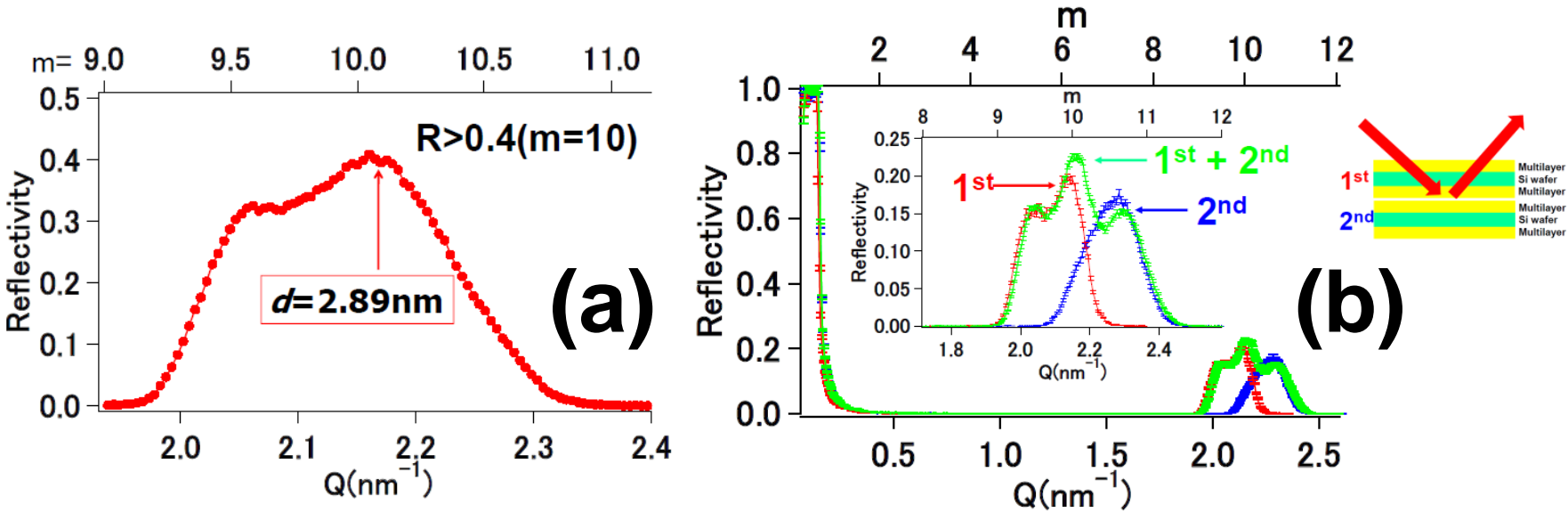

Fig. 3 Reflectivity of NiC/Ti stacking mirrors in with the following number of layers: (a) 85060; and (b) 10929(1 $\left.1^{\text {st }}\right)$, $10336\left(2^{\text {nd }}\right)$, and $21265\left(1^{\text {st }}+2^{\text {nd }}\right)$, respectively.

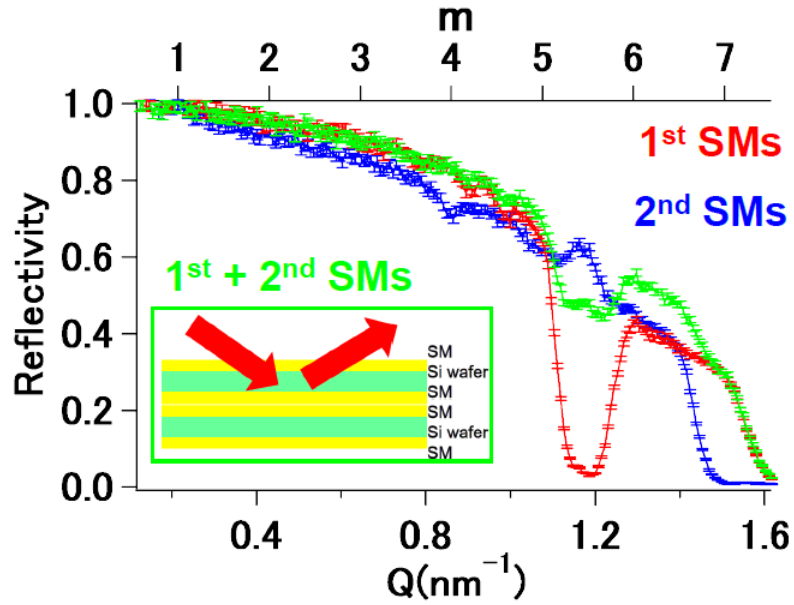

Fig. 4 Reflectivity of $m=7 \mathrm{NiC} / \mathrm{Ti}$ supermirror stacking two mirrors, as shown in the insert.

(a)

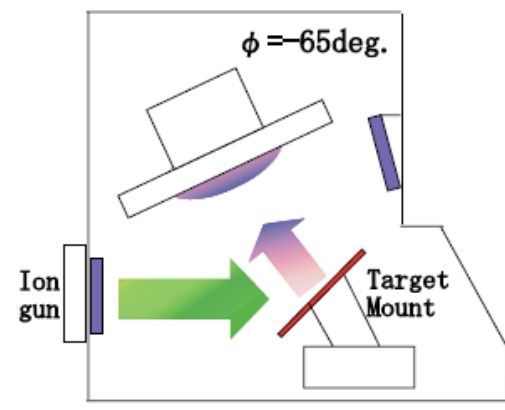

(b)

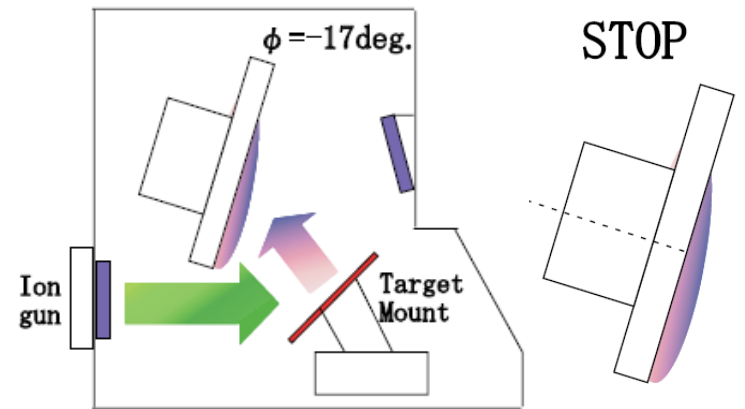

(c)

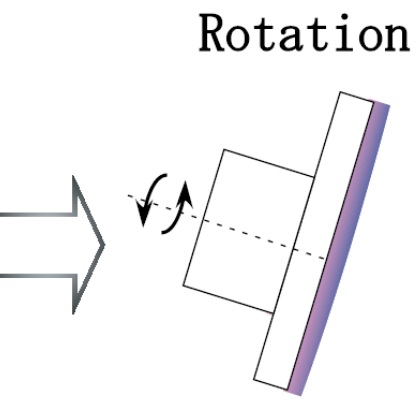

Fig. 5 Schematic illustration of thickness dependence on the tilt angle for (a) small, and (b) large samples in the case of stopping the substrate holder; (c) a schematic image of thickness dependence by rotating the substrate holder 

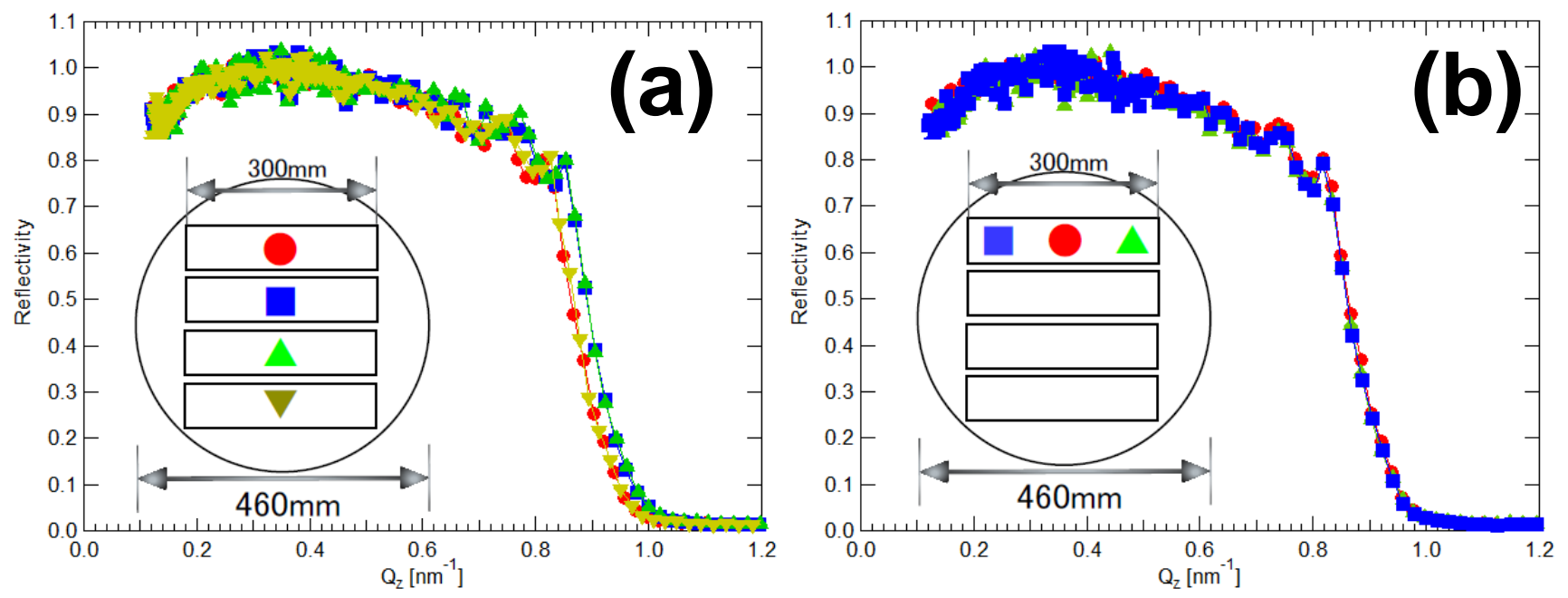

201 Fig. 6 Reflectivities of $m=4 \mathrm{NiC} / \mathrm{Ti}$ supermirror with (a) the center of four mirrors, and (b) the left, center, and right of 202 top mirror.
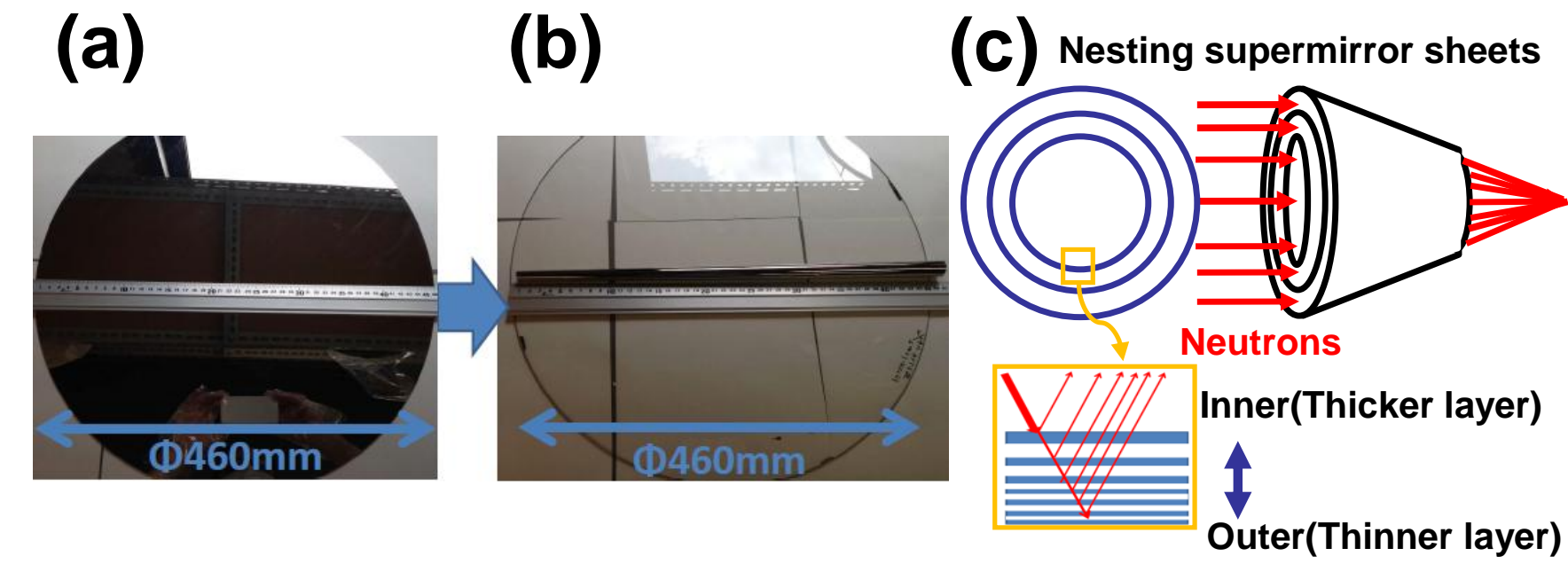

204 Fig. 7 Photograph of (a) $m=5 \mathrm{NiC} / \mathrm{Ti}$ supermirror deposited on glass substrate and (b) the $m=5 \mathrm{NiC} / \mathrm{Ti}$ self-standing 205 supermirror peeling off from the glass substrate. (c) Schematic view of strong focusing device based on the nested 206 axisymmetric supermirror sheets. 


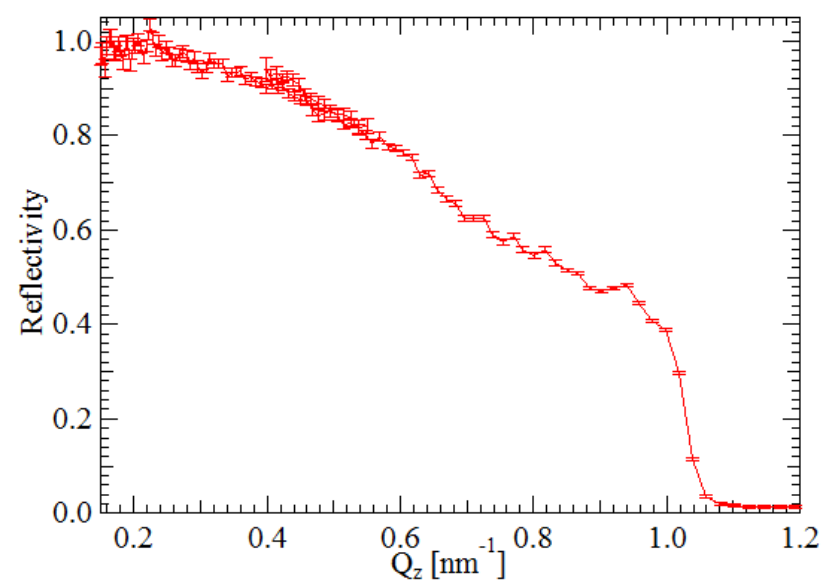

209 Fig. 8 Reflectivities of the $m=5$ NiC/Ti self-standing supermirror sheet affixed on glass plate.

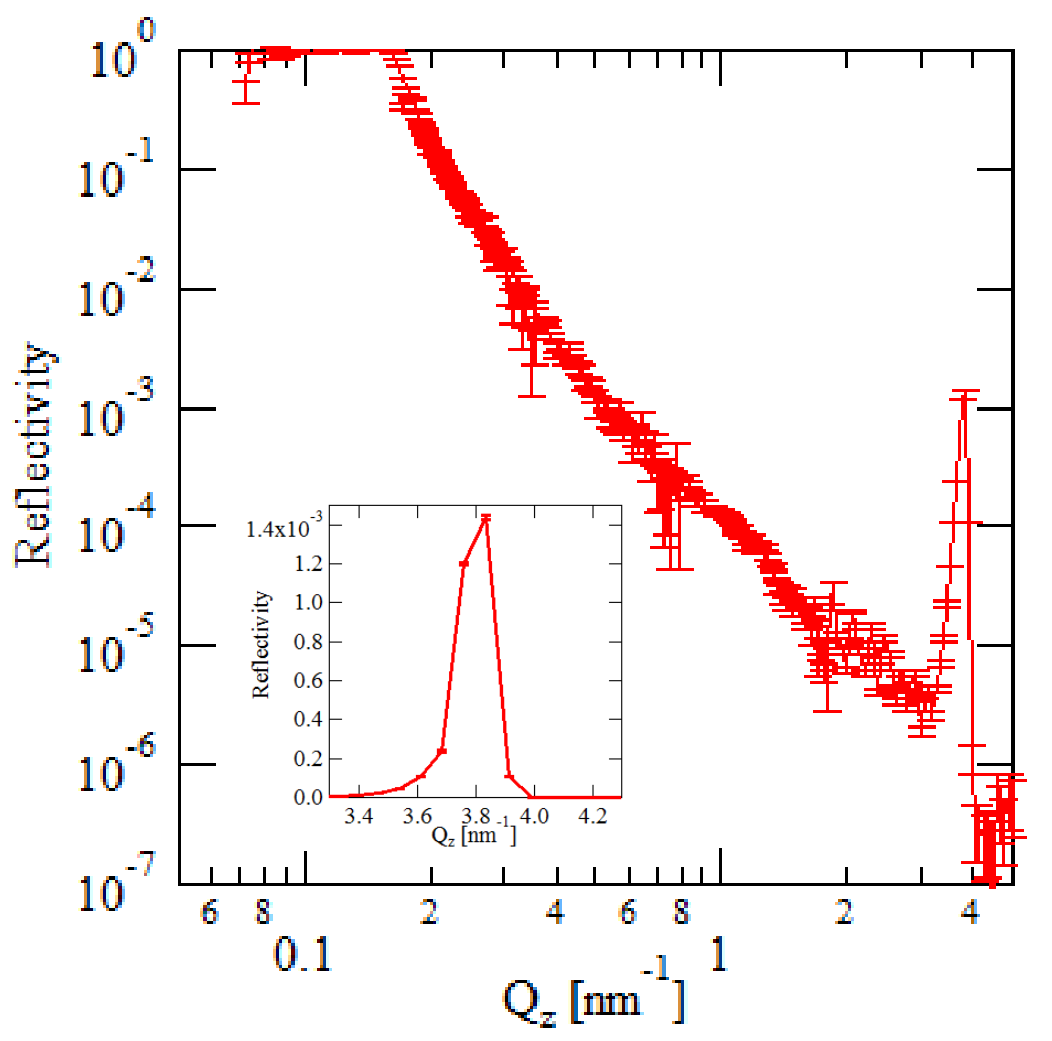

211 Fig.9 Reflectivity of $d=1.65 \mathrm{~nm} \mathrm{NiC/Ti}$ self-standing multilayer mirror in which layers numbered 10000 . The insert 212 shows an enlarged view at $3.3<\mathrm{Q}_{\mathrm{z}}<4.3(\mathrm{~nm})$.

\section{Acknowledgments}

215 The authors gratefully acknowledge Dr. Thomas Krist and his group in HZB for use of the TEM image. This work was 216 supported by a Grant-in-Aid for Scientific Research, Grant Nos. 23360428 of JSPS, program of Development of 217 System and Technology for Advanced Measurement Analysis (SENTAN), JST, the Neutron Scattering Program 
Advisory Committee of IMSS, KEK (Proposal Nos. 2009S07, 2009S08), and the Photon and Quantum Basic Research Coordinated Development Program from the Ministry of Education, Culture, Sports and Technology, Japan. The basic KUR-IBS system was installed in the NOP project (Special Coordination Funds for Promoting Science and Technology of the Ministry of Education of Japanese Government).

\section{References}

224 [1] http://www.ucans.org/, Physics Procedia Vol.26 (2012) and Vol. 60( 2014).

[2] F. Mezei, Comm. Phys., 1(1976)81.

[3] T.Akiyoshi, T.Ebisawa, T.Kawai, F.Yoshida, M.Ono, S.Tasaki, S.Mitani, T.Kobayash, S.Okamoto, J.Nucl.Sci.Tech. 29(1992) 939

228 [4] S.Tasaki,, T.Ebisawa, T.Akiyoshi, T.kawai, Nucl. Instrum. Methods Phys. Res. A 501 (1995) 501.

[5] M. Hino, H. Sunohara, Y.Yoshimura, R.Maruyama, S.Tasaki, H.Yoshino, Y.Kawabata, Nucl. Instrum. Methods Phys. Res. A 574 (2004) 54.

231 [6] R. Maruyama, D. Yamazai, T. Ebisawa, M. Hino, and K. Soyama, Physica, B 385-386 (2006) 1256.

232 [7] R. Maruyama, D. Yamazai, T. Ebisawa, M. Hino, and K. Soyama, Thin Solid Films 515 (2007) 5704.

233 [8] R. Maruyama, D. Yamazai, T. Ebisawa, K. Soyama, Nucl. Instrum. Methods Phys. Res. A 600 (2009) 68.

234 [9] R. Maruyama, D. Yamazai, T. Ebisawa, K. Soyama, J. Appl. Phys.105 (2009), 083527.

[10] K.Yamamura, M.Nagano, N.Zettsu, D.Yamazaki, R.Maruyama, K.Soyama, Nucl. Instrum. Methods Phys. Res. A 616 (2010) 193-196.

[11] T. Krist, A.Teichert, R.Kovács-Mezei, L.Rosta, Springer Series in optical science, Vol.137 (Modern Developments in X-Ray and Neutron Optics), (2008) 355.

[12] http://www.swissneutronics.ch/

[13] R. Kova'cs-Mezei,T. Krist, Zs. Re`vay, Nucl. Instrum. Methods Phys. Res. A 586 (2008) 51.

[14] M. Senthil Kumar, P. Böni, M. Horisberger, Nucl. Instrum. Methods Phys. Res. A 574 (2004) 90.

[15] K Ikeda,T Adachi,T Shinohara,T Morishima, K Hirota, Y Takizawa, H Sato, H.M Shimizu, M Hino, S Tasaki, T Oku, K Soyama, Nucl. Instrum. Methods Phys. Res. A 574 (2004) 78.

[16] M. Aya, C.Schanzer, M. Wolff, J. Stahna, Nucl. Instrum. Methods Phys. Res. A 562 (2006) 389.

[17] A. Biswas, R. Sampathkumar, Ajaya Kumar, D. Bhattacharyya, N. K. Sahoo, K. D. Lagoo, R. D. Veerapur, M. Padmanabhan, R. K. Puri, Debarati Bhattacharya, Surendra Singh, S. Basu, Rev.Sci. Instrum. 85, (2014)123103.

[18] M.Hino, M.Kitaguchi, H.Hayashida, S.Tasaki, T.Ebisawa, R.Maruyama, N.Achiwa, Y.Kawabata, Nucl. Instrum. Methods Phys. Res. A 600 (2009) 207.

[19] T. Oda, M. Hino, N. L. Yamada, et al., to be published in J.Nucl. Sci. Technol.

[20] K Mishima, S.Imajo, M.Hino, T.Ino, Y.Iwashita, R.Katayama, M.Kitaguchi, T.Oda, H.M.Shimizu, M.Utsuro, S.Yamashita, T.Yoshioka , J. Phys.: Conf. Ser.528(2014) 012030.

[21] N. K. Pleshanov, Nucl. Instrum. Methods Phys. Res. A524 (2004) 273.

[22] N. L. Yamada, N. Torikai, K. Mitamura, H. Sagehashi, S. Sato, H. Seto, T. Sugita, S. Goko, M. Furusaka, T. Oda, M. Hino, T. Fujiwara, H. Takahashi, A. Takahara, Euro. Phys. J. Plus 126 (2011), 108.

[23] K. Mitamura, N. L. Yamada, H. Sagehashi, N. Torikai, H. Arita, M. Terada, M. Kobayashi, S. Sato, H. Seto, S. Gokou, 
256 M. Furusaka, T. Oda, M. Hino, H. Jinnai and Atsushi Takahara, Polymer J. 100 (2013), 45.

257 [24] K. Yamamura, M. Nagano, H. Takai, N. Zettsu, D. Yamazaki, R. Maruyama, K. Soyama, and S. Shimada, Optics express 17 (2009) $258 \quad 6414$

259 [25] J. Guo, S. Takeda, S. Morita, M. Hino, T. Oda, J. Kato, Y. Yamagata, M. Furusaka, Optics express 22 (2014) 24666.

260 [26] C. Schanzer, P. Böni and M. Schneider, J. Phys: Conf. Ser. 251, (2010) 012082.

261 [27] A. Steyerl, H. Nagel, F.-X. Schreiber, K.-A. Steinhauser, R. Gähler, W. Gläser, P. Ageron, J.M. Astruc, W. Drexel, R. 262 Gervais, W. Mampe, Phys. Lett. A 116 (1986) 347.

263 [28] Y.Kawabata, M.Suzuki, S.Tasaki, K.Somemiya, Nucl. Instrum. Methods Phys. Res. A420 (1999) 213. 